\title{
Evolution of Compatibility Range in the Rice-Magnaporthe oryzae System: An Uneven Distribution of R Genes Between Rice Subspecies
}

\author{
Romain Gallet, Colin Fontaine, François Bonnot, Joëlle Milazzo, Christophe Tertois, Henri Adreit, \\ Virginie Ravigné, Elisabeth Fournier, and Didier Tharreau
}

First and eighth authors: INRA, UMR BGPI, F-34398 Montpellier, France; second author: Centre d'Ecologie et des Sciences de la Conservation-UMR 7204, Muséum national d'Histoire naturelle, Paris, France; third, fourth, fifth, sixth, seventh, and ninth authors: CIRAD, UMR BGPI, F-34398 Montpellier, France; and seventh author: CIRAD, UMR PVBMT, F-97410 Saint-Pierre, France.

Accepted for publication 10 December 2015.

\begin{abstract}
Gallet, R., Fontaine, C., Bonnot, F., Milazzo, J., Tertois, C., Adreit, H., Ravigné, V., Fournier, E., and Tharreau, D. 2016. Evolution of compatibility range in the rice-Magnaporthe oryzae system: An uneven distribution of $\mathrm{R}$ genes between rice subspecies. Phytopathology 106:348-354.

Efficient strategies for limiting the impact of pathogens on crops require a good understanding of the factors underlying the evolution of compatibility range for the pathogens and host plants, i.e., the set of host genotypes that a particular pathogen genotype can infect and the set of pathogen genotypes that can infect a particular host genotype. Until now, little is known about the evolutionary and ecological factors driving compatibility ranges in systems implicating crop plants. We studied the evolution of host and pathogen compatibility ranges for rice blast disease,
\end{abstract}

ABSTRACT which is caused by the ascomycete Magnaporthe oryzae. We challenged 61 rice varieties from three rice subspecies with 31 strains of $M$. oryzae collected worldwide from all major known genetic groups. We determined the compatibility range of each plant variety and pathogen genotype and the severity of each plant-pathogen interaction. Compatibility ranges differed between rice subspecies, with the most resistant subspecies selecting for pathogens with broader compatibility ranges and the least resistant subspecies selecting for pathogens with narrower compatibility ranges. These results are consistent with a nested distribution of $\mathrm{R}$ genes between rice subspecies.

Additional keywords: generalist/specialist trade-off, infection severity, nestedness, Oryza sativa, specialization.
Plant pathogens pose a major threat to agriculture, and their emergence on new crop varieties may occur in two different ways (Parker and Gilbert 2004). The first is an extension of host compatibility range (see definitions in the Materials and Methods section) for pathogens jumping from one host species (or genotype) to a new one (De Vienne et al. 2009). The second operates at the intraspecific level and involves the accidental introduction of an allopatric pathogen genotype that occurs to be highly pathogenic on local plants (Barrès et al. 2008; Fontaine et al. 2013; Garbelotto et al. 2013). Understanding the factors underpinning the evolution of compatibility range in host plants and pathogens could permit a better prediction and control of pathogen emergences.

The molecular (i.e., proximate) determinants of host-pathogen compatibility have been extensively studied. In addition to nonspecific host defense mechanisms (PAMP-triggered immunity), host-pathogen compatibility has been shown to be tightly related to host-pathogen recognition systems (Jones and Dangl 2006), through the well-known complete resistance mechanism. In this gene-forgene model (Flor 1956), the ability of a plant to prevent a pathogen from causing an infection is governed by resistance $(\mathrm{R})$ genes, which encode proteins recognizing pathogen effectors (encoded by "avirulence" genes). When the product of the $\mathrm{R}$ gene recognizes the pathogen effector, a hypersensitive response (HR) is triggered, blocking the entry of the pathogen into the plant. In addition to this complete resistance mechanism, the specificity of host-pathogen

Corresponding author: R. Gallet; E-mail address: rgallet@gmail.com

*The $\boldsymbol{e}$-Xtra logo stands for "electronic extra" and indicates that four supplementary figures and one supplementary table are published online.

http://dx.doi.org/10.1094/PHYTO-07-15-0169-R

(C) 2016 The American Phytopathological Society interactions is modulated by a second, less well understood mechanism of plant resistance known as partial or quantitative resistance (Poland et al. 2009). Hosts displaying partial resistance are infected, but display less severe damage than susceptible plants. Partial resistance is probably determined by disparate molecular mechanisms, including nonspecific resistance mechanisms (e.g., thicker cell walls, the production of chitinase or detoxifying enzymes, see Fukuoka et al. [2009] and Parker and Gilbert [2004]) and partial breakdown of the resistance conferred by R genes (Brodny et al. 1986; Li et al. 1999; Nass et al. 1981; Poland et al. 2009). However, it remains unclear whether this last phenomenon is general or limited to a few $\mathrm{R}$ genes. If overcoming $\mathrm{R}$ genes are frequently involved in partial resistance in plants, then we would expect a positive correlation between complete and partial resistance. Plants with few $\mathrm{R}$ genes would show very low partial resistance and would display broad compatibility range, while plants with many R genes would be expected to have a narrow compatibility range and a high level of partial resistance. By contrast, if partial resistance is mediated principally by nonspecific resistance mechanisms (e.g., thicker cell walls, etc.), there should be no correlation unless there is a trade-off between these traits, but we are not aware of such a constraint in rice or other species. To our knowledge, such correlation has never been looked at in rice.

The eco-evolutionary (i.e., ultimate) determinants of host-pathogen compatibility have also been studied. Evolutionary factors, such as local adaptation trade-offs (Agudelo-Romero et al. 2008; Wallis et al. 2008) or the phylogenetic distance between plant species (De Vienne et al. 2009; Gilbert and Webb 2007; Ness et al. 2011; Poulin et al. 2011; Schulze-Lefert and Panstruga 2011), and ecological factors, such as the spatial distribution of host populations and the rates of dispersal of the host and pathogen (Thrall and Burdon 2002), have been shown to influence host-pathogen compatibility.

Despite the large body of work on both molecular and ecoevolutionary determinants of host-pathogen compatibility range, 
some results remain elusive. For instance, many cross-inoculation experiments reported that some pathogens could infect more plant genotypes than the number of genotypes to which they were exposed to, i.e., that their fundamental compatibility range was larger than their realized compatibility range (De Vienne et al. 2009, and references therein). Some studies also observed particular local adaptation patterns (hereafter called the asymmetric pattern of compatibility range) in which highly resistant plant genotypes (specialists with narrow compatibility range) were associated with generalist pathogens (showing wide compatibility ranges), and reciprocally less resistant plant genotypes (generalists) were associated with pathogens showing narrow compatibility ranges (specialists) (De Vienne et al. 2009; Thrall and Burdon 2003). At the interspecific level using several species of Microbotryum and Caryophyllaceae, De Vienne et al. (2009) found that both host and pathogen phylogenies were good predictors of compatibility range. They hypothesized that the asymmetric pattern of compatibility range they observed, was resulting from hot spots and cold spots of coevolution (De Vienne et al. 2009). Thrall and Burdon (2003) made similar observations at the intraspecific level with the Linum marginale-Melampsora lini system. They showed that $\mathrm{R}$ genes were the main molecular driver of compatibility range, and that the gene-for-gene coevolutionary process between resistance genes in plants and avirulence genes in the pathogen was responsible of the asymmetry in compatibility range. Plant genotypes with many $\mathrm{R}$ genes (specialists) were selecting generalist fungal pathogens able to overcome all these resistances, while plant genotypes with few $\mathrm{R}$ genes (generalists) were selecting specialist pathogens. Whether a phylogenetic effect (De Vienne et al. 2009) could explain this observation at the intraspecific level could not be verified for the following reasons: (i) Thrall and Burdon (2003) work is anterior to De Vienne et al. (2009), and (ii) Thrall and Burdon (2003) studied natural populations in which the genotypic composition was unknown. Thus, both of these studies provided key information to explain the origin of the asymmetry in compatibility range, even though the generality of this phenomenon remains to be proven by studying other plant-pathogen systems.

In this work, we aimed at establishing whether $\mathrm{R}$ genes could be responsible for the strong phylogenetic signal of compatibility ranges as observed by De Vienne et al. (2009). Specifically, we tested the impact of $\mathrm{R}$ gene distribution in rice varieties on the structure of the interaction network with various genotypes of the blast pathogen at the intraspecific level (Fig. 1). Rice blast, caused by the ascomycete fungus Magnaporthe oryzae, is a good system to address the effect of $\mathrm{R}$ gene distribution on the evolution of host and pathogen compatibility ranges. First, the rice species is composed of several subspecies, which phylogenetic history is well known (Huang et al. 2012). Secondly, the worldwide population genetic structure of $M$. oryzae has recently been described and its relation with the history of the rice-M. oryzae system has been established (Saleh et al. 2014). Thirdly, the molecular determinants of rice compatibility range have been studied extensively (Ballini et al. 2008; Vergne et al. 2008), and differential varieties (varieties for which R gene contents are known) containing specific R genes are available, allowing testing the relationship between compatibility ranges and $\mathrm{R}$ gene contents.

We investigated the factors underlying the evolution of compatibility range with the rice- $M$. oryzae system, by characterizing rice- $M$. oryzae compatibility range fully, at the scale of the rice species. We carried out a large-scale cross-inoculation experiment, in which we assessed the ability of 31 fungal strains to infect 61 rice varieties. All fungal strains and 38 varieties were chosen so as to represent the genetic clusters of $M$. oryzae that have spread around the world (japonica and indica) and the three main rice subspecies (indica, temperate japonica, and tropical japonica). The other 23 varieties were differential varieties. We used these latter varieties to estimate the number of R genes that each fungal strain could overcome.

\section{MATERIALS AND METHODS}

Definitions. Compatibility range. For pathogens, compatibility range represents the proportion of host genotypes that a particular pathogen genotype can infect (i.e., the average infection success of the fungal strain). Reciprocally, host's compatibility range represents the proportion of pathogen genotypes that can infect a particular host genotype.

Severity score. This score evaluates the severity of infection, following the inoculation of rice plants with a $M$. oryzae strain.
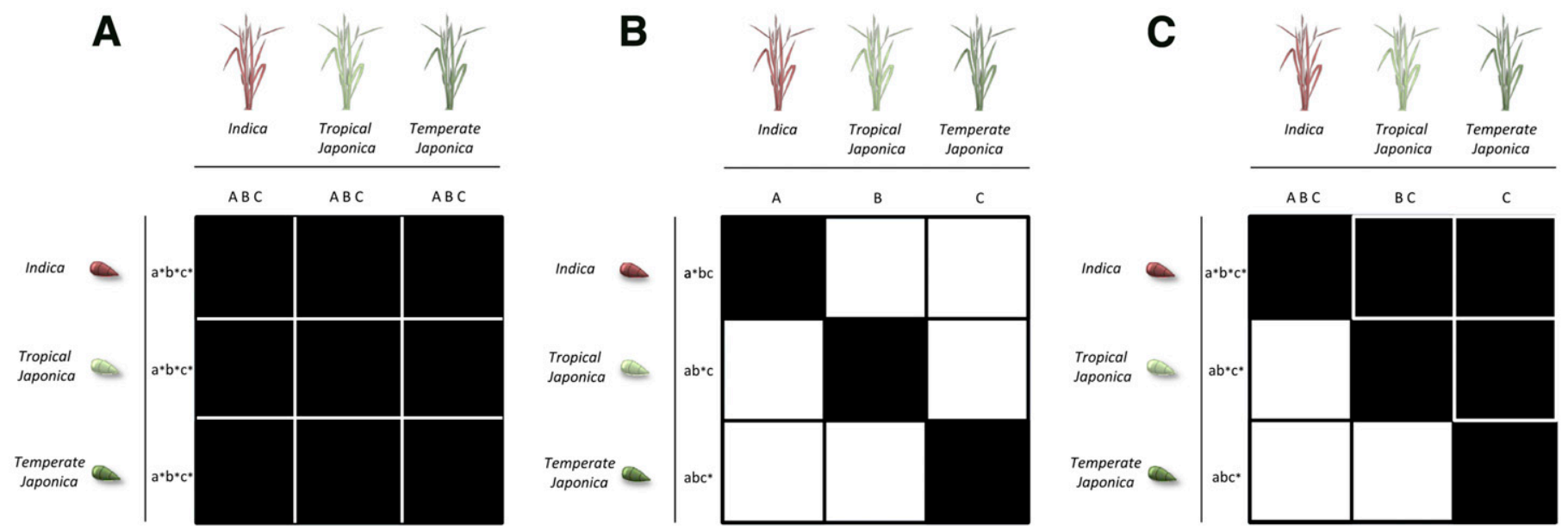

Fig. 1. Different interaction patterns between host variety groups (rice) and pathogen genetic groups (Magnaporthe oryzae) in a gene-for-gene framework, under several hypotheses. A, All variety groups contain the same set of $\mathrm{R}$ genes. Under this hypothesis, for the pathogen, adapting to the local host is equivalent to adapting to all host variety groups. B, Each variety group contains a unique set of $\mathrm{R}$ genes (the sets of $\mathrm{R}$ genes present differ between variety groups). In this case, overcoming the resistance of the sympatric (local) host does not enable the pathogen to infect allopatric (foreign) hosts. C, The temperate japonica variety group has only some of the R genes carried by the tropical japonica variety group, and the tropical japonica variety group has only some of the R genes carried by the indica variety group. In this case, indica strains can overcome all $\mathrm{R}$ genes and can therefore infect all varieties, whereas tropical japonica strains can infect only japonica variety groups, and temperate japonica strains can infect only temperate japonica varieties. Columns correspond to hosts, and lines correspond to pathogens. Black squares represent compatible interactions (i.e., the pathogen can infect the host), whereas white squares represent incompatible interactions (no infection). The capital letters above each column represent $\mathrm{R}$ genes, and the lowercase letters next to each line represent $a v r$ genes. Stars above $a v r$ genes represent mutations enabling the pathogen to escape recognition by the product of the $\mathrm{R}$ gene. 
Infection severity was estimated on 20 to 25 plants, according to a scale extending from 1 to 6 (Silué et al. 1992). A score of 1 indicates unsuccessful infection, and a score of 2 indicates HR-controlled infection (both of which are considered to correspond to complete resistance). Scores of 3 to 6 indicate successful infections of increasing severities (partial resistance). Each score describes a specific stage of lesion formation: 1 = no lesion (unsuccessful infection), 2 = black dots (infection controlled by the HR response), $3=$ round lesions with differentiated centers less than $1 \mathrm{~mm}$ in diameter, $4=$ round lesions with differentiated centers more than $1 \mathrm{~mm}$ in diameter, $5=$ typical diamond-shaped susceptible lesions with a brown margin, and $6=$ typical diamond-shaped susceptible lesions with no brown margin (Supplementary Fig. S1). Severity score does not provide an estimate of aggressiveness, as it does not take into account the number of lesions on the plant. Severity score simply reflects the "immunological" state of the plant, and the extent to which it is able to control the infection. Scores from 3 to 6 provide an estimate of infection severity (i.e., of partial resistance).

Studied rice varieties and pathogen strains. Two experiments were performed in this study. In the local adaptation experiment, the rice varieties and fungal strains were chosen so as to represent the three main rice subspecies (indica, temperate japonica, and tropical japonica; Supplementary Fig. S2) and the two genetic clusters of $M$. oryzae that have spread worldwide (i.e., clusters B and C, referred to hereafter as the japonica and indica clusters, respectively) (Saleh et al. 2014). Most of the rice accessions (other than differentials) were chosen to be, as much as possible, representative of the genetic diversity of the main rice subgroups. In a core collection containing mainly traditional varieties (Garris et al. 2005), we selected randomly a given number of varieties in the three main subgroups. We used a sample of 31 fungal strains: 17 indica strains, collected from indica rice varieties; 14 japonica strains collected from japonica rice varieties (genetic cluster B), 10 of which were collected from temperate japonica rice varieties, the other four being collected from tropical japonica rice varieties (Saleh et al. 2014). We used these strains to challenge 38 rice varieties: 22 indica, 8 temperate japonica, and 8 tropical japonica. In a second experiment aiming at evaluating the number of $\mathrm{R}$ genes overcome by the fungal strains used in the first experiment, we used the 31 fungal strains described above to inoculate 23 differential varieties with known resistance genes (differentials; listed in Supplementary Table S1).

Cross-inoculation experiments. Design. The two experiments were performed at the same time. All rice varieties were inoculated with all fungal strains, following the protocol described by Sallaud et al. (2003) and illustrated in Supplementary Fig. S3. Briefly, rice seeds were sown in peaty soil in plastic trays and plants were grown in the greenhouse until the four to five leaf stage. The 61 varieties were grown in four trays, with 16 varieties per tray $(16 \times$ $4=64$ but three varieties were excluded from the analysis because their origins were not clear). For each variety, we obtained 20 to 25 plants. Plants were inoculated by spraying $30 \mathrm{ml}$ of spore suspension/tray. The suspension contained 25,000 spores $/ \mathrm{ml}$ and was supplemented with $0.5 \%$ gelatin. At each date, six to eight independent inoculations were performed (an inoculation corresponding to the spraying of a particular strain on all 61 varieties). We always inoculated all 61 varieties together, to avoid complex "date $\times$ variety" effects. In this experiment, the experimental unit corresponds to a group of 20 to 25 plants of a specific variety, inoculated with a specific strain (e.g., M. oryzae A $\times$ rice variety 1$)$. Each experimental unit was replicated two to three times, at different dates. The complete design required the inclusion of 20 inoculation dates. Symptoms were scored seven days after inoculation (one score per experimental unit, i.e., per group of 20 to 25 inoculated plants).

We measured the host and pathogen compatibility ranges, respectively, by determining the proportion of host varieties that could be infected with a particular fungal strain/group, and the proportion of fungal strains capable of infecting a particular plant variety/group of varieties. We initially pooled lesions in two categories: incompatible interactions (lesions with severity scores of 1 and 2 , see the scale description above), and compatible interactions (lesion with severity scores of 3 to 6). We then calculated the proportion of compatible interactions by dividing the number of compatible interactions by the total number of crosses, for each rice or $M$. oryzae genotype. We also calculated the mean severity score for a given plant variety, for successful infections (severity scores of 3 to 6).

Statistics. Inoculation onto cultivated rice varieties. For the local adaptation analysis, the 38 plant varieties sampled worldwide were inoculated with the 31 fungal strains. The success of infection (used to estimate compatibility ranges), which is a binary response (infection or no infection) was analyzed using a generalized linear model with a binomial error structure and logit link function, including rice variety and fungal strain as main effects, and the variety-strain interaction. Contrasts were used to assess the specific degrees of freedom of main effects and interactions.

Inoculation onto differential rice varieties. We analyzed the number of R genes overcome by $M$. oryzae strains, by inoculating a subset of 23 differential varieties of known $R$ gene content with the 31 fungal strains. We considered $19 \mathrm{R}$ genes in this study (some varieties carried two resistance genes and some genes were present in several different varieties). When a given pathogen strain was able to infect the variety considered (i.e., a compatible interaction was observed), we considered the strain to be able to overcome all the $\mathrm{R}$ genes carried by the variety. After the inoculation of all differential varieties with a particular fungal strain, we could determine the total number of $\mathrm{R}$ genes overcome by this strain. In total, we used 1426 interactions (31 strains $\times 23$ differential varieties $\times 2$ replicates). We used a generalized linear model with a binomial error structure and logit link function, including fungal strain group as a main effect, to analyze these data.

Nestedness computation and significance. A network is considered as nested if the host or pathogen compatibility range of species/ individuals is a subset of the host or pathogen compatibility range of more generalist species/individuals. Nestedness was estimated by calculating the nestedness metric based on overlap and decreasing fill (NODF) index developed by Almeida-Neto et al. (2008). This index relates to the proportion of interactions common to species pairs over the network and has been shown to have good statistical properties.

We determined whether the nestedness value obtained for our dataset was significant, by comparing it with those of interaction matrices generated under a null model (mgen null model in bipartite package of $\mathrm{R}$ software). In the null model used, the probability of an interaction between a pathogen strain and the rice variety tested is proportional to the product of compatibility range, such that strains/ varieties with high compatibility range have a higher probability of being assigned to an interaction than strains/varieties with low compatibility range. Such a null model thus conserves the heterogeneity of compatibility range observed in the original data set.

\section{RESULTS}

Local adaptation and nested model of interaction. We determined the compatibility range (proportion of compatible interactions) of the various groups of $M$. oryzae strains with the three rice subspecies (Fig. 2). We found significant effects of plant variety group, fungal strain group and the interaction between these factors (Table 1). The plant variety group effect was due to temperate japonica varieties being susceptible to a larger number of strains (wider compatibility range) than the indica and tropical japonica varieties (contrast: temperate japonica versus indica + tropical japonica $z$-value $\left.=10.894, P<2 \times 10^{-16}\right)$, for which compatibility ranges were similar (contrast: indica versus tropical japonica $z$-value $=-0.156, P=0.876$ ). The strain group effect could be explained by two main observations: (i) the indica strains had the 
highest infection frequencies on hosts of any group, and (ii) the temperate japonica strains had the lowest infection frequencies on indica and tropical japonica plants. Finally, the host plant group $\times$ fungal strain group interaction could be explained by observations consistent with local adaptation patterns: temperate japonica strains mostly infected temperate japonica varieties, tropical japonica strains infected tropical japonica varieties more frequently than indica varieties and indica strains infected indica varieties more frequently than tropical japonica varieties (contrast $z$-value $=2.839, P=0.005$ ) .

Most indica strains acted as generalists, infecting most varieties. Tropical japonica strains were able to infect japonica varieties (tropical and temperate), and temperate japonica strains were essentially specialists, with most strains able to infect only temperate japonica varieties. This pattern is consistent with a nested pattern of interaction (Fig. 1C). We performed a nestedness analysis on this dataset (Fig. 3) to test this hypothesis. The observed nestedness score was significantly higher than the one expected under the null model, demonstrating significant nestedness of the matrix (Fig. 3B).

Importantly, the interaction matrix is overall nested, with narrow ranges being subsets of wider ranges. This nestedness was also true for strain groups with indica, tropical japonica, and temperate japonica strains showing nested wide, intermediate, and narrow compatibility ranges, respectively (Fig. 3C). However, nestedness was not so clearly correlated with variety group. Temperate japonica varieties showed a significantly larger compatibility ranges than varieties from the two other subspecies (Figs. 2 and 3C). But, compatibility ranges of indica and tropical japonica rice subspecies were highly variable among varieties and not significantly different (Figs. 2 and 3 C). It is to be noted that, as there is an interaction in our statistical analysis between the two factors "rice subspecies" and "strain group" (interaction observable on Figure 2), the statistical results shown in Figure 3C should be taken with care. However, it summarizes quite well the matrix shown in Figure $3 \mathrm{~A}$, and also allows a comparison of our results with those from Thrall and Burdon (2003).

The implication of $\mathbf{R}$ genes. The compatibility range of M. oryzae strains and the proportion of $\mathrm{R}$ genes they could overcome were highly correlated $(t=2.8918, \mathrm{df}=29, P$ value $=0.00719 ;$ Fig. $4 \mathrm{~A})$.

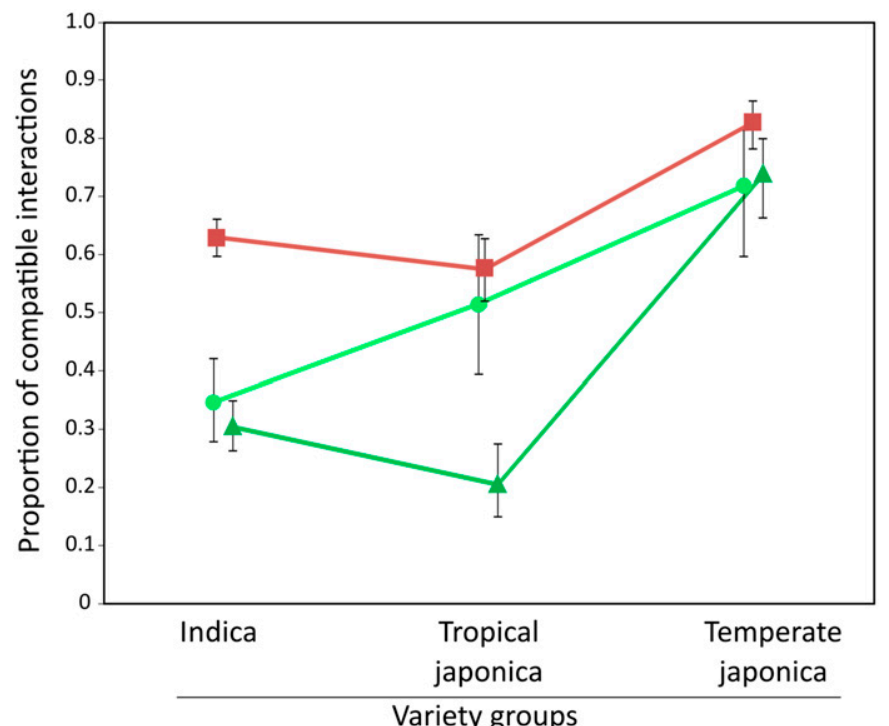

Fig. 2. Proportion of compatible interactions between the various fungal strain groups and the different rice categories. The three lines on the graph represent the host compatibility ranges (proportion of compatible interactions) for the three Magnaporthe oryzae categories (indica: line with square symbols; tropical japonica, line with round symbols; and temperate japonica, line with triangular symbols) on the three categories of rice plants (indica, tropical japonica, and temperate japonica). Error bars were calculated with the general linear model used to analyze the data and represent the $95 \%$ confidence intervals. The curves have been shifted laterally to improve readability.
Fungal strain groups were able to overcome different numbers of $\mathrm{R}$ genes ( $\mathrm{df}=2, P=0.002$; Fig. $4 \mathrm{~B})$. This result was mostly driven by the fact that temperate japonica strains were able to overcome fewer $\mathrm{R}$ genes than tropical japonica and indica strains (contrast indica and tropical japonica versus temperate japonica: $t=3.059, P=$ 0.0023; Fig. 4B). Surprisingly, indica and tropical japonica strains were able to overcome the same proportion of $\mathrm{R}$ genes, whereas these strains show significantly different compatibility ranges. This discrepancy could be due a lack of statistical power related to the fact that we used a limited number of strains. The fungal strain $\times R$ gene matrix showed a similar pattern to that of the matrix shown in Figure 3, even though the ranking of fungal strains was modified, and the matrix not significantly nested.

Relationship between the pathogen compatibility range and infection severity. We investigated the relationship between the number of strains to which varieties were susceptible (the pathogen compatibility range of the host) and the ability of these varieties to control infections (mean severity score of successful infections). There was a positive correlation between the number of fungal strains capable of infecting a given variety and the severity of infection $\left(t=5.5562, \mathrm{df}=62, P=6.17 \times 10^{-7} ;\right.$ Fig. 5$)$. Thus, varieties able to protect themselves against infection by many different strains also seem to be able to limit the severity of the infection when it does occur, whereas varieties infected by many strains also displayed more severe infection.

\section{DISCUSSION}

A nested pattern of compatibility. The confrontation of 31 M. oryzae strains representative of the worldwide diversity against a sample of 38 rice varieties resulted in a nested pattern of compatibility strongly structured by the pathogen strain group. Indica, tropical japonica and temperate japonica strains had respectively wide, intermediate and narrow compatibility ranges. Strains sampled on indica can thus be described as generalists able to infect most rice varieties, whatever their subspecies and geographical origin. Strains sampled on tropical japonica varieties were able to infect as many tropical and temperate japonica varieties but fewer indica varieties than strains isolated on indica varieties. Finally, strains from temperate japonica varieties were able to infect almost the same number of temperate japonica varieties but fewer indica and tropical japonica varieties than the others strains, making them specialists of temperate japonica varieties.

The nested pattern of compatibility range was also apparent for rice varieties, but less clearly. Because the compatibility ranges of strains was clearly structured by host subspecies, we would have expected varieties belonging to the indica, tropical and temperate japonica subspecies to have respectively narrow, intermediate, and wide compatibility ranges (like in Thrall and Burdon [2003]). We did observe that on average, temperate japonica varieties had wider compatibility ranges than the two other subspecies (Fig. 3C). However, indica and tropical japonica varieties had similar average compatibility ranges and important variance (Fig. 3C).

Yet it is quite clear that temperate japonica varieties can be infected by a greater number of strains than both indica and tropical japonica varieties, and this, independently of strain group. This pattern may result from the ecological constraints in the areas where the varieties are grown. Temperate japonica varieties are mainly cultivated in temperate areas and under irrigated conditions in which

TABLE 1. General linear model analysis for the local adaptation experiment

\begin{tabular}{lrrrcr}
\hline & df & Deviance & Residual df & Residual deviation & $P\left(>\chi^{2}\right)$ \\
\hline Null & & & 2,564 & $3,526.7$ & \\
Rice & 2 & 167.409 & 2,562 & $3,359.3$ & $<2.2 \mathrm{E}-16$ \\
Fungus & 2 & 190.754 & 2,560 & $3,168.6$ & $<2.2 \mathrm{E}-16$ \\
Rice $\times$ fungus & 4 & 23.277 & 2,556 & $3,145.3$ & 0.00011 \\
\hline
\end{tabular}


epidemics are usually less frequent and less severe than in tropical areas. Resistance to blast is therefore less of a concern to rice breeders that tend to prioritize improving yield and gustative characters over resistance during rice selection. Resistance to a wide range of strains could then be lost, either incidentally, i.e., because of the genetic drift inherent to breeding process, or because resistance mechanisms trade off with positively selected characteristics.

Under this "drifting" scenario, narrow compatibility ranges would be an ancestral state for rice varieties, and wide compatibility ranges would rather be a derived state. However, compatibility range distribution is also highly variable within indica and tropical japonica subspecies. Both of these subspecies comprise varieties with very wide and very narrow compatibility ranges. In indica subspecies, 10 out of the 12 varieties showed the narrowest compatibility range, and 5 out of the 10 varieties showed the widest compatibility ranges. Such variability suggests that compatibility range of varieties is not fully constrained by their phylogenetic history and that it may respond rapidly to environmental or agronomic (breeding) constraints. Both subspecies are cultivated over very large spatial scales in a diversity of eco-climatic conditions. It is thus possible that in some localities or under some conditions, resistance to pathogens is not prioritized in the breeding process, and varieties with wide compatibility ranges are selected for by rice breeders.

$\mathbf{R}$ genes are involved in compatibility range. Challenging $M$. oryzae strains with differential rice varieties for which $\mathrm{R}$ gene contents are known permitted us to observe a correlation between the compatibility range of fungal strains and their ability to overcome $\mathrm{R}$ genes (Fig. 4). Such correlation is a clear indication that $\mathrm{R}$ genes are major drivers of compatibility range in the rice blast disease. This result confirms the implication of $\mathrm{R}$ genes in compatibility ranges already observed on rice (Divya et al. 2014; Hittalmani et al. 2000). This result also suggests that the nested pattern of compatibility ranges we observed can be interpreted in terms of R genes. First, the nested pattern implies that the network of compatibility between rice and $M$. oryzae is made of a single compartment (Fig. 1C, and not like $1 \mathrm{~B}$ where there are several independent compartments), i.e., that all rice subspecies share the same pool of R genes. In other words, the "phylogenetic" effect detected in our local adaptation experiment could be explained by the fact that temperate japonica varieties have fewer R genes than the other subspecies. Second, generalist pathogen strains (with a wider compatibility range) would be able to overcome more $\mathrm{R}$ genes than specialist strains only able to overcome a few $\mathrm{R}$ genes.

Interestingly, some results of the present study remain puzzling. First, we observed that even if the compatibility range of pathogens and their ability to overcome R gene correlate positively (Fig. 4A), indica and tropical japonica strains have different compatibility ranges (Fig. 3C) but could overcome the same proportion of $\mathrm{R}$ genes (Fig. 4B). We hypothesize that this discrepancy could be due to the fact that rice varieties used in the two experiments (with
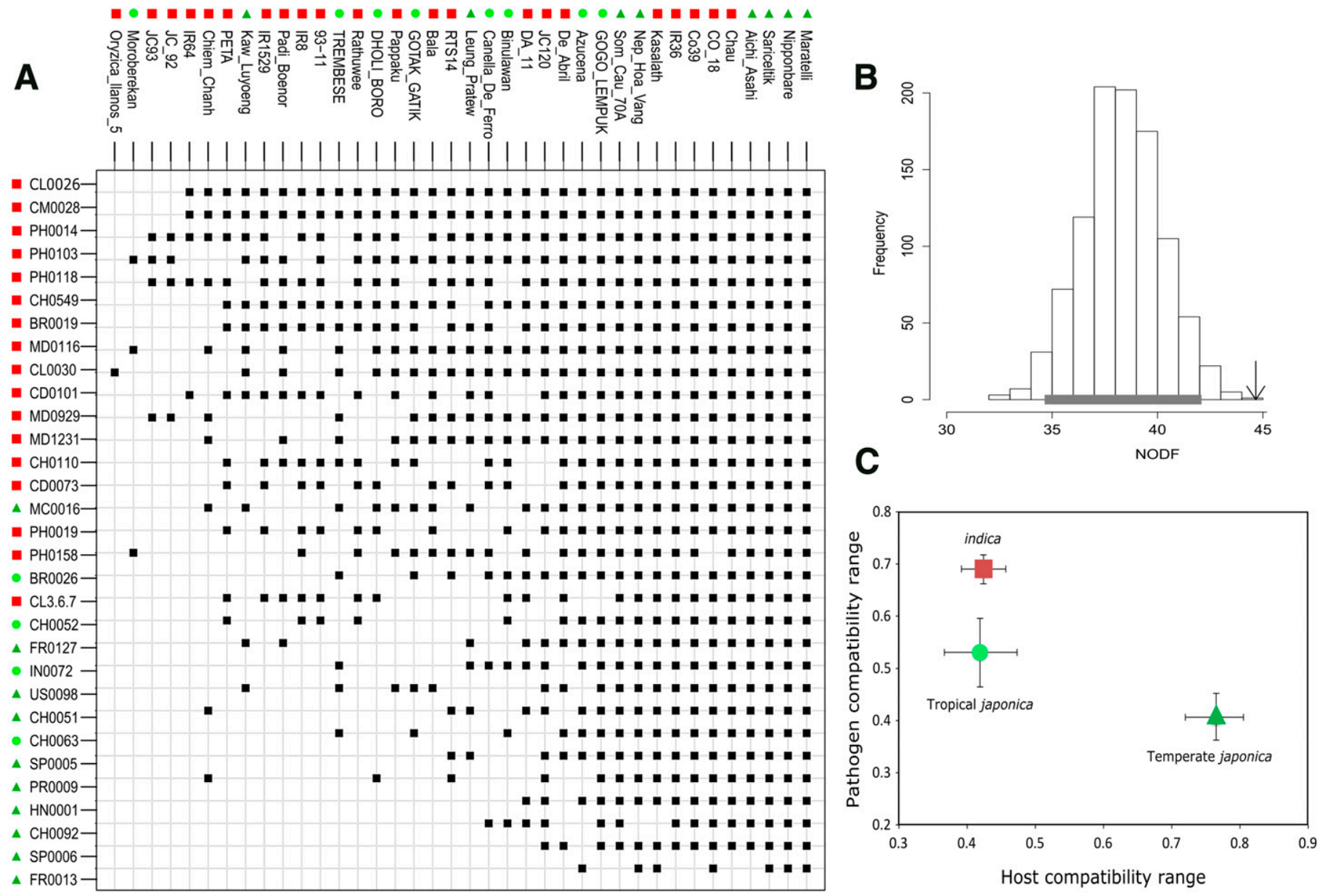

Fig. 3. Matrix showing the interaction between rice and Magnaporthe oryzae. A, The actual interaction matrix between rice varieties (column) and M. oryzae strains (lines) obtained in our experiments. Black squares indicate compatible infections. Individual strains and varieties are ordered according to their contribution to NODF. indica rice varieties or fungal strains sampled from indica varieties are represented by squares, tropical japonica varieties and the strains sampled from them are indicated by circles, and temperate japonica varieties and the strains sampled from them are represented by triangles. B, The distribution of nestedness values (NODF) of 1,000 matrices generated with the null model, and the nestedness value of the observed dataset (arrow). The observed interaction matrix is significantly nested, as its NODF value is greater than $97.5 \%$ of the null expectation (represented by a gray rectangle). C, Both host and pathogen compatibility ranges for each group (indica, tropical japonica, and temperate japonica). Error bars represent $95 \%$ confidence intervals, but see discussion on this statistical analysis. 
differential and nondifferential varieties) did not contain the same sets of $\mathrm{R}$ genes. For instance, if some $\mathrm{R}$ genes often present in indica varieties were absent in the differential varieties, we could not observe that those fungal strains sampled on indica plants could overcome more $\mathrm{R}$ genes than those sampled on tropical japonica. Another explanation would be that some $\mathrm{R}$ genes or $\mathrm{R}$ gene combinations are more frequent in some subspecies than others.

Second, indica and tropical japonica varieties have the same average compatibility range and share the same set of $\mathrm{R}$ genes (Fig. $3 \mathrm{~A}$ and $\mathrm{C}$ ), a result that is not congruent with the local adaptation of fungal strains of the rice subspecies on which they were sampled (Fig. 2). The fact that some $\mathrm{R}$ genes or $\mathrm{R}$ gene combinations were more frequent in some rice subspecies would be enough to create a local adaptation pattern. Another possibility is that fungal pathogens also adapt to other plant factors than $\mathrm{R}$ gene products. A firm demonstration of the present results will therefore require additional work to exhaustively describe the $\mathrm{R}$ gene content of varieties representative of the three rice subspecies.

The non-negligible relationship between $R$ genes and infection severity. Severity scores allowed estimating both compatibility range and the severity of successful infections. This was a good opportunity to verify if the partial breakdown of R gene-mediated resistance is a general trend or simply restricted to a few $\mathrm{R}$ genes. More generally, it also permits study of the relationship between these two traits, which is equivalent to studying the relationship
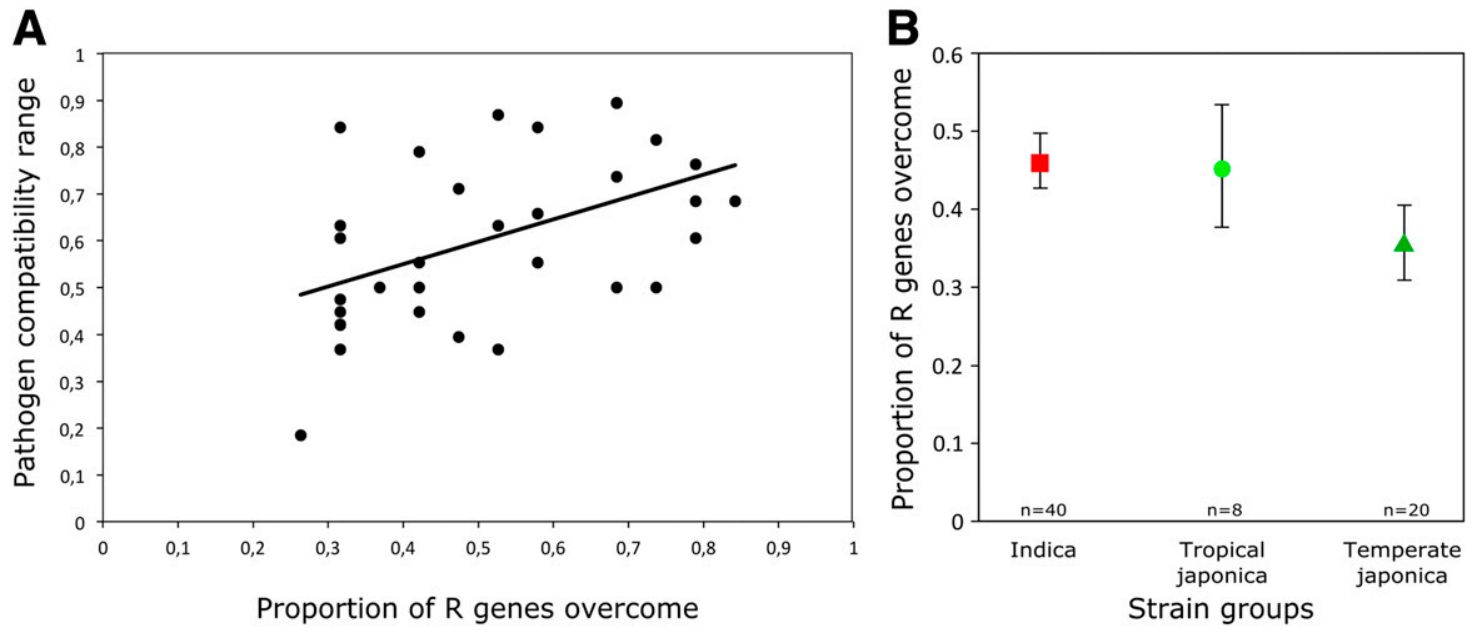

Fig. 4. Magnaporthe oryzae strains and $\mathrm{R}$ genes. Proportion of $\mathrm{R}$ genes overcome by $M$. oryzae strains, and the different strain groups of $M$. oryzae. A, The correlation between the compatibility range and the proportion of $\mathrm{R}$ genes overcome for each strain used in our local adaptation experiment $\left(n=31, R^{2}=0.22, t=\right.$ $2.8918, \mathrm{df}=29, P$ value $=0.00719)$. B, The proportion of the $19 \mathrm{R}$ genes overcome by the strain groups, estimated with a generalized linear model. The error bars show the confidence intervals for each estimate. The numbers at the bottom of the graph show the numbers of data points used to obtain these estimates (two or three replicates were used).

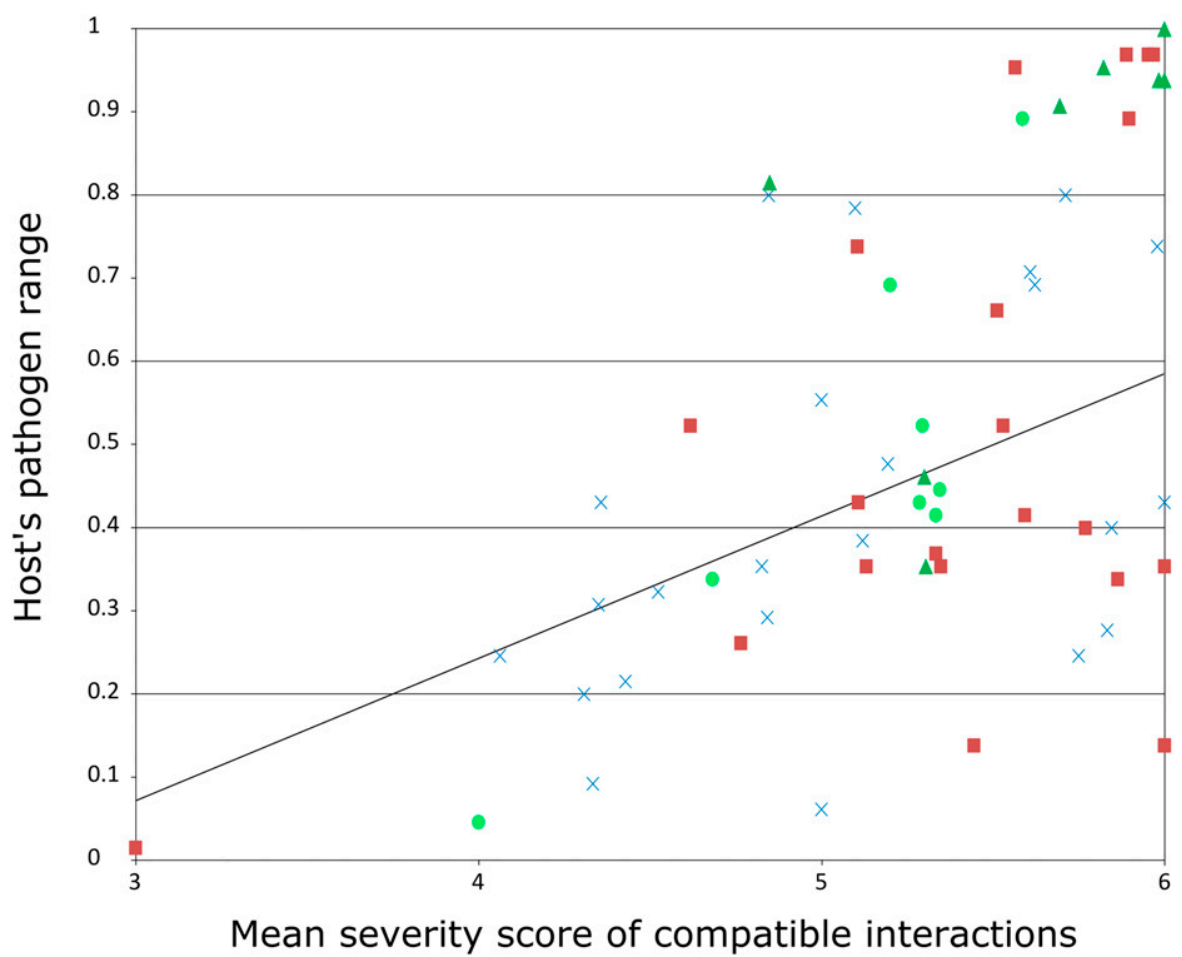

Fig. 5. Plot showing the pathogen compatibility range of the host against the mean severity score of compatible interactions for individual varieties. The indica varieties are represented as squares, tropical japonica varieties are shown as circles, temperate japonica varieties as triangles, and unassigned plant varieties are represented as crosses. The regression line is plotted. Pearson's correlation, calculated for the whole data set, was significant $\left(t=4.63, \mathrm{df}=59, P=2.052 \times 10^{-5}\right)$. 
between complete (infection/no infection) and partial resistance (the ability to control fungal growth within the plant).

We found a positive correlation between susceptibility and pathogen compatibility range in rice varieties (Fig. 5). The rice varieties attacked by the largest numbers of fungal strains and thus with the broadest compatibility ranges, were unable to control $M$. oryzae growth within the plant. Conversely, varieties with a narrow compatibility range were able to efficiently control the growth of the infecting pathogen. This correlation is consistent with the hypothesis that in rice, $\mathrm{R}$ genes are involved in the genetic control of both partial and complete resistance (Ballini et al. 2008). This implication of $\mathrm{R}$ genes in partial resistance does not exclude the possibility of other partial resistance mechanisms occurring in rice.

The correlation between mean severity score and compatibility range also provides valuable information about the potential benefits of introducing more $\mathrm{R}$ genes into rice varieties. First, it shows that some varieties are resistant to most pathogen genotypes. This may be due to these rice varieties having large numbers of $\mathrm{R}$ genes, or to the presence of a few broad-spectrum $\mathrm{R}$ genes. Second, rice varieties resistant to most pathogen genotypes (i.e., with a narrow compatibility range) suffered only mild symptoms. This association between narrow pathogen compatibility range and low susceptibility indicates that, even if an M. oryzae strain can initiate infection in such plants, the plant is able to control its subsequent growth (and thus a potential outbreak). A trade-off between the ability to overcome $\mathrm{R}$ genes and spore production in M. oryzae may explain this pattern (Thrall and Burdon 2003). A clear demonstration of the existence of such a tradeoff in $M$. oryzae would confirm the value of introducing additional $\mathrm{R}$ genes into rice, provided that the addition of such genes is not costly to the plant. A few M. oryzae genotypes might still be able to infect such improved plants, but they would not thrive in these plants and would be unable to start an epidemic. Further investigations are required into the costs of accumulating $\mathrm{R}$ genes in plants and of the breakdown of resistance by $M$. oryzae.

Conclusion. In this work, we found that interactions between rice and $M$. oryzae are structured in a nested way. This pattern was consistent with a nested distribution of $\mathrm{R}$ genes among rice subspecies, which caused nested levels of compatibility ranges between rice subspecies and the asymmetric pattern of compatibility range. These results were mostly driven by the temperate japonica varieties having wider compatibility range than indica and tropical japonica varieties. We hypothesize that a nested distribution of $\mathrm{R}$ genes between plant species could explain why phylogeny has been shown to be a good predictor of infection success (De Vienne et al. 2009).

Our results might also provide an explanation to the frequent observation of potential compatibility range of pathogens being larger than their observed compatibility range. Pathogens adapting to host plants carrying many R genes, will have a large compatibility range and therefore will be able to infect all plant genotypes that carry the same set (or a subset) of these $\mathrm{R}$ genes, regardless of a primary exposure of the pathogen to these plant genotypes. The host shifts of pathogen often observed in nature might actually not correspond to any sort of shift for the pathogen, if the $\mathrm{R}$ gene contents in the different plant species are the same.

\section{ACKNOWLEDGMENTS}

We thank J. B. Morel and anonymous reviewers for fruitful comments on the manuscript, and G. Thebaud for his help on specific statistical analyses.

\section{LITERATURE CITED}

Agudelo-Romero, P., de la Iglesia, F., and Elena, S. F. 2008. The pleiotropic cost of host-specialization in Tobacco etch potyvirus. Infect. Genet. Evol. 8:806-814.

Almeida-Neto, M., Guimaraes, P. R., Loyola, R. D., and Werner, U. 2008. A consistent metric for nestedness analysis in ecological systems: Reconciling concept and measurement. Oikos 117:1227-1239.

Ballini, E., Morel, J.-B., Droc, G., Price, A., Courtois, B., Notteghem, J.-L., and Tharreau, D. 2008. A genome-wide meta-analysis of rice blast resistance genes and quantitative trait loci provides new insights into partial and complete resistance. Mol. Plant-Microbe Interact. 21:859-868.

Barrès, B., Halkett, F., Dutech, C., Andrieux, A., Pinon, J., and Frey, P. 2008. Genetic structure of the poplar rust fungus Melampsora larici-populina: Evidence for isolation by distance in Europe and recent founder effects overseas. Infect. Genet. Evol. 8:577-587.

Brodny, U., Nelson, R. R., and Gregory, L. V. 1986. The residual and interactive expression of "defeated" wheat stem rust resistance genes. Phytopathology 76:546-549.

De Vienne, D. M., Hood, M. E., and Giraud, T. 2009. Phylogenetic determinants of potential host shifts in fungal pathogens. J. Evol. Biol. 22:2532-2541.

Divya, B., Robin, S., Rabindran, R., Senthil, S., Raveendran, M., and Joel, J. 2014. Marker assisted backcross breeding approach to improve blast resistance in Indian rice (Oryza sativa) variety ADT43. Euphytica 200:61-77.

Flor, H. H. 1956. The complementary genetic systems in flax and flax rust. Adv. Genet. 8:29-54.

Fontaine, M. C., Gladieux, P., Hood, M. E., and Giraud, T. 2013. History of the invasion of the anther smut pathogen on Silene latifolia in North America. New Phytol. 198:946-956.

Fukuoka, S., Saka, N., Koga, H., Ono, K., Shimizu, T., Ebana, K., Hayashi, N., Takahashi, A., Hirochika, H., Okuno, K., and Yano, M. 2009. Loss of function of a proline-containing protein confers durable disease resistance in rice. Science 325:998-1001.

Garbelotto, M., Guglielmo, F., Mascheretti, S., Croucher, P. J. P., and Gonthier, P. 2013. Population genetic analyses provide insights on the introduction pathway and spread patterns of the North American forest pathogen Heterobasidion irregulare in Italy. Mol. Ecol. 22:4855-4869.

Garris, A. J., Tai, T. H., Coburn, J., Kresovich, S., and McCouch, S. 2005. Genetic structure and diversity in Oryza sativa L. Genetics 169:1631-1638.

Gilbert, G. S., and Webb, C. O. 2007. Phylogenetic signal in plant pathogenhost range. Proc. Natl. Acad. Sci. USA 104:4979-4983.

Hittalmani, S., Parco, A., Mew, T. V., Zeigler, R. S., and Huang, N. 2000. Fine mapping and DNA marker-assisted pyramiding of the three major genes for blast resistance in rice. Theor. Appl. Genet. 100:1121-1128.

Huang, X., Kurata, N., Wei, X., Wang, Z.-X., Wang, A., Zhao, Q. 2012. A map of rice genome variation reveals the origin of cultivated rice. Nature 490:497-501.

Jones, J. D. G., and Dangl, J. L. 2006. The plant immune system. Nature 444: 323-329.

Li, Z.-K., Luo, L. J., Mei, H. W., Paterson, H., Zhao, X. H., and Zhong, D. B. 1999. A "defeated" rice resistance gene acts as a QTL against a virulent strain of Xanthomonas oryzae pv. oryzae. Mol. Gen. Genet. 261:58-63.

Nass, H. A., Pedersen, W. L., MacKenzie, D. R., and Nelson, R. R. 1981. The residual effects of some "defeated" powdery mildew resistance gene in isolines of winter wheat. Phytopathology 71:1315-1318.

Ness, J. H., Rollinson, E. J., and Whitney, K. D. 2011. Phylogenetic distance can predict susceptibility to attack by natural enemies. Oikos 120:1327-1334.

Parker, I. M., and Gilbert, G. S. 2004. The evolutionary ecology of novel plantpathogen interactions. Annu. Rev. Ecol. Evol. Syst. 35:675-700.

Poland, J. A., Balint-Kurti, P. J., Wisser, R. J., Pratt, R. C., and Nelson, R. J. 2009. Shades of gray: The world of quantitative disease resistance. Trends Plant Sci. 14:21-29.

Poulin, R., Krasnov, B. R., and Mouillot, D. 2011. Host specificity in phylogenetic and geographic space. Trends Parasitol. 27:355-361.

Saleh, D., Milazzo, J., Adreit, H., Fournier, E., and Tharreau, D. 2014. SouthEast Asia is the center of origin, diversity and dispersion of the rice blast fungus, Magnaporthe oryzae. New Phytol. 201:1440-1456.

Sallaud, C., Lorieux, M., Roumen, E., Tharreau, D., Berruyer, R., and Svestasrani, P. 2003. Identification of five new blast resistance genes in the highly blast-resistant rice variety IR64 using a QTL mapping strategy. Theor. Appl. Genet. 106:794-803.

Schulze-Lefert, P., and Panstruga, R. 2011. A molecular evolutionary concept connecting nonhost resistance, pathogen host range, and pathogen speciation. Trends Plant Sci. 16:117-125.

Silué, D., Notteghem, J.-L., and Tharreau, D. 1992. Evidence of a gene-forgene relationship in the Oryza sativa-Magnaporthe grisea pathosystem. Phytopathology 82:577-580.

Thrall, P. H., and Burdon, J. J. 2002. Evolution of gene-for-gene systems in metapopulations: The effect of spatial scale of host and pathogen dispersal. Plant Pathol. 51:169-184.

Thrall, P. H., and Burdon, J. J. 2003. Evolution of virulence in a plant host-pathogen metapopulation. Science 299:1735-1737.

Vergne, E., Ballini, E., Droc, G., Tharreau, D., Nottéghem, J.-L., and Morel, J.-B. 2008. ARCHIPELAGO: A dedicated resource for exploiting past, present, and future genomic data on disease resistance regulation in rice. Mol. Plant-Microbe Interact. 21:869-878.

Wallis, C. M., Stone, A. L., Sherman, D. J., Damsteegt, V. D., Gildow, F. E., and Schneider, W. L. 2008. Adaptation of Plum pox virus to a herbaceous host (Pisum sativum) following serial passages. J. Gen. Virol. 88:28392845. 